\title{
ESTUDO DA ANSIEDADE COMO VARIÁVEL NO RELACIONAMENTO ALUNO-PACIENTE
}

Maria Lúcia Araújo Sadala*

O estudo tem o objetivo de analisar a ansiedade como variável na comunicação de alunas de graduação em enfermagem ao prestarem assistência aos pacientes. Os dados foram obtidos pela pesquisadora através da observação participante, estratégia que se constituiu simultaneamente de três procedimentos: registro das interações aluno-paciente pela pesquisadora, registro das mesmas pelos alunos e entrevistas dos alunos pela pesquisadora imediatamente após os dois primeiros procedimentos. A população da pesquisa constituiu-se de 10 alunos de enfermagem, acompanhados durante atividades de estágios curriculares nos $3^{\circ}, 4^{\circ}$ e $6^{\circ}$ semestre do curso. A ansiedade, tanto dos alunos como dos pacientes, aparece como variável interferindo no uso da comunicação terapêutica pelos alunos.

UNITERMOS: ansiedade - comunicação terapêutica

\section{INTRODUÇÃO}

Durante os últimos cinco anos temos observado com particular interesse as manifestações de ansiedade dos alunos de graduação em enfermagem em situações de estágio supervisionado, através de seus relatos e trabalhos desenvolvidos nas disciplinas Relacionamento Enfermeira-Paciente I, II e III. Os alunos da Turma de 1989 foram acompanhados pela docente das referidas disciplinas em três períodos, $3^{\circ}, 4^{\circ}$ e $6^{\circ}$ períodos do curso de graduação, durante atividades de assistência aos pacientes, com o objetivo de estudar o desenvolvimento da habilidade de comunicação (SADALA, 1992). Os resultados deste trabalho chamaram nossa atenção para a ansiedade que aparece como elemento importante nas interações analisadas, emergindo então a proposta de um estudo que enfoque a ansiedade como variável relevante no relacionamento aluno-paciente.

\footnotetext{
* Docente Responsável pela Disciplina Relacionamento Enfermeira-Paciente no Curso de Enfermagem da Faculdade de Medicina de Botucatu, UNESP
} 
O estresse e a ansiedade tem sido reconhecidos atualmente como um problema profissional de importância fundamental para as enfermeiras. A responsabilidade e o esforço em providenciar assistência a pessoas em situação crítica exigem muito do profissional, física e emocionalmente. Particularmente em áreas que exigem maior empenho e competência dos profissionais: unidades intensivas, pediatria, pacientes terminais, os dois problemas acima referidos aparecem em níveis consideráveis (COLLIGAN et al. 1977; MARSHAL, 1980; BAILEY, 1985).

Em estudos realizados com estudantes de enfermagem, a ansiedade e referida como uma forma de estresse que aparece ao desenvolverem atividades práticas para as quais não se sentem preparados $(\mathrm{BIRCH}, 1979)$. Isto é intensificado quando as estudantes prestam assistência a crianças, doentes em estado grave, pacientes terminais (BAILEY, 1985). A ansiedade e o estresse são associados pelos estudantes a sua inexperiência, ao despreparo em lidar com situações críticas e a interferência das horas exigidas para estudos teóricos. MENZIES (1960), na década de 60, num estudo precursor sobre a ansiedade da enfermeira, atribui a intenção de abandono do curso por estudantes de enfermagem ao estresse da profissão.

Nos trabalhos citados, os termos ansiedade e estresse são utilizados aparentemente como sinônimos. MAY (1980), diferencia estresse e ansiedade. Para este autor, estresse e um termo fisiológico: consiste em parte da situação de ameaça. A ansiedade é como manobramos o estresse, portanto a nossa reação global a situação de ameaça.

PESSOTTI (1978) define o estresse como o componente nuclear da ansiedade, sendo compreendido como as condições aversivas que provocam comportamentos de esquiva ou fuga. A ansiedade, por outro lado, abrange três significados básicos: a ansiedade implica algum modo de impotência do indivíduo, ocorre a instauração de uma condição aversiva ou penosa, e algum grau de incerteza ou dúvida. O estresse para este autor estaria, portanto, contido no conceito de ansiedade. Na literatura conceitos diversos sobre o termo são encontrados, no geral distinguindo o estresse da ansiedade.

Utilizaremos neste estudo o conceito de ansiedade significando um sentimento de apreensão difusa. Ela difere do conceito de medo: o medo e uma reação a um perigo específico, concreto; ao passo que a ansiedade é não específica, é vaga e sem objeto (MAY, 1980). A ansiedade tem um propósito: proteger-nos dos perigos que ameaçam a nossa existência ou os nossos valores. Ela é útil, portanto, para a sobrevivência e para o nosso desenvolvimento, desde que mantida em níveis suportáveis, quando atua como um estímulo a consciência, a vigilância e a ação (MAY, 1980).

Manter a ansiedade sob controle, possibilitando perceber a situarão real, refletir sobre ela e procurar as soluções mais adequadas dentro das nossas possibilidades - eis o objetivo que a teoria da comunicação terapêutica propõe para possibilitar uma comunicação 
efetiva com o paciente (STEFANELLI, 1985). A enfermeira dispõe dos recursos propostos por esta teoria: estratégias que estimulam o paciente a expressar-se e as medidas terapêuticas, basicamente o oferecimento de apoio, as quais utilizadas em conjunto contribuem para baixar os níveis de ansiedade do paciente, e conseqüentemente os da profissional.

Para HOFLING (1970) a enfermeira, ao perceber a ansiedade do paciente, experimentara um aumento no nível da sua própria ansiedade, o que a estimulará a procurar ajudar o paciente a resolver o seu problema, resultando na redução da ansiedade de ambos. Caso contrário, a ansiedade dos dois tenderá a aumentar.

Com base nos conceitos expostos nos parágrafos anteriores, procuramos neste trabalho estudar como a ansiedade aparece na prática dos alunos ao desenvolverem atividades de enfermagem e, nessas situações, como ela se apresenta frente à utilização da comunicação terapêutica.

A análise da comunicação desenvolvida pelos estudantes nas suas interações com os pacientes será feita com o referencial teórico de EDWARDS e BRILHART \(1985) que analisa a compreensão dos sinais não-verbais e a sua função na comunicação e o de STEFANELLI (1985) que descreve a teoria da comunicação terapêutica e a sua utilização como habilidade e competência na enfermagem; ambos utilizados em trabalhos de investigação por SILVA (1989) e SADALA (1992).

\section{OBJETIVOS}

Identificar a ansiedade manifestada pelos alunos de graduação em enfermagem durante interações com o paciente.

Identificar a percepção que os alunos têm da própria ansiedade e da ansiedade do paciente.

Analisar como a ansiedade interfere no uso da comunicação terapêutica pelo aluno, ao relacionar-se com o paciente.

\section{METODOLOGIA}

Trata-se de uma pesquisa exploratório-descritiva. Utilizou-se como método de coleta de dados a observação participante (LUDKE, 1986), a pesquisadora no papel de observador como participante combinando simultaneamente os seguintes procedimentos: observação direta e registro das interações aluno-paciente durante atividades de assistência; relato 
escrito das mesmas pelos alunos logo após as interações; e entrevista com os alunos pela pesquisadora, imediatamente após os dois primeiros procedimentos, com o objetivo de clarificar e validar os dados obtidos através da observação e registro das interações.

A pesquisadora acompanhou um grupo de 10 alunos do curso de graduação em enfermagem, durante atividades de assistência ao paciente, em três fases: durante o estágio curricular de Fundamentos de Enfermagem ( $3^{\circ}$ período letivo), durante o estágio curricular de Enfermagem Médico-Cirúrgica ( $4^{\circ}$ período letivo) e durante o estágio curricular de Enfermagem Ginecológica ( $6^{\circ}$ período letivo).

A análise dos dados obtidos através dos procedimentos descritos fundamentou-se nos conceitos de referencial teórico descrito na página 03, a partir dos quais foi feita a classificação dos mesmos em categorias descritivas (LUDKE, 1986), após leituras sucessivas do material até se chegar aos seus elementos componentes, sem perder de vista a relação desses elementos com os outros componentes, ou seja, com o objeto estudado como um todo.

\section{ANÁLISE E DISCUSSÃO DOS DADOS}

Os dados apresentados a seguir, sob a forma de tabelas, foram extraídos dos procedimentos propostos: observação das comunicações alunos-pacientes; dos relatos dessas comunicações feitos pelos alunos; e da análise das entrevistas com os alunos logo após as interações.

A Tabela 1, que descreve os sentimentos manifestados não verbalmente pelos alunos e percebidos pela pesquisadora, é apresentada a seguir:

TABELA I - SENTIMENTOS DOS ALUNOS PERCEBIDOS PELA PESQUISADORA, NAS INTERAÇÕES ENTRE OS ALUNOS E OS PACIENTES. Botucatu, 1992

\begin{tabular}{|c|c|c|c|c|}
\hline \multicolumn{5}{|c|}{ FASES } \\
\hline SENTIMENTOS & $1^{a}$ & $2^{a}$ & $3^{a}$ & TOTAL \\
\hline Ansiedade & 22 & 16 & 32 & 70 \\
\hline Atenção & 52 & 55 & 86 & 193 \\
\hline Desconsideração & 22 & 15 & 12 & 38 \\
\hline Determinação & 39 & 55 & 69 & 163 \\
\hline Dúvida & 19 & 14 & 25 & 58 \\
\hline Indignação & 2 & - & - & 2 \\
\hline Resignação & - & 2 & - & 2 \\
\hline Satisfação & 7 & - & 7 & 14 \\
\hline Surpresa & 2 & 1 & 1 & 4 \\
\hline Tristeza & - & 3 & 1 & 4 \\
\hline TOTAL & 165 & 161 & 233 & 559 \\
\hline
\end{tabular}


Podemos observar que os sentimentos percebidos em maior número pela pesquisadora foram atenção, determinação, ansiedade e dúvida.

A ansiedade e a dúvida aparecem mais vezes na $3^{a}$ fase, quando se poderia esperar que diminuíssem, devido à experiência já vivenciada pelos alunos quando chegam ao $3^{\circ}$ ano do curso. No entanto, as interações desta fase, foram desenvolvidas na clínica ginecológica. A ansiedade das pacientes era intensa por não serem informadas do seu diagnóstico (na maioria dos casos, suspeitas de câncer de mama ou de útero); perguntavam aos alunos, de modo direto ou indireto, sobre isso e eles não tinham condições de responder, ou por ignorarem o diagnóstico, e por não poderem fornecê-lo as pacientes. Isto tudo resultando em ansiedade e dúvida manifestadas não verbalmente pelos alunos, e validadas pela pesquisadora através dos outros procedimentos metodológicos.

A satisfação, a tristeza, a surpresa, a indignação e a resignação apareceram em situações nas quais observamos o desenvolvimento de empatia entre o aluno e o paciente.

Os sentimentos percebidos pela pesquisadora foram confirmados pelos alunos nos seus relatos escritos e nas entrevistas, que foram realizados imediatamente após as interações. De qualquer modo, é importante ressaltar que todas as interpretações foram feitas no contexto das interações, e somente neste contexto podem ser analisadas e validadas com informações dos próprios alunos.

TABELA 2 - DISTRIBUIÇÃO DOS MODOS DE COMUNICAÇÃO UTILIZADOS PELOS ALUNOS DURANTE AS INTERAÇÕES COM OS PACIENTES. Botucatu, 1992

\begin{tabular}{lcccc}
\hline \multicolumn{5}{c}{ FASES } \\
\hline MODOS DE COMUNICAÇÃo & $\mathbf{1}^{\mathbf{a}}$ & $\mathbf{2}^{\mathbf{a}}$ & $\mathbf{3}^{\mathbf{a}}$ & TOTAL $^{\text {TO }}$ \\
\hline Confirmação & 10 & 28 & 41 & 79 \\
Negação & 05 & 9 & 6 & 20 \\
Desconfirmação & 12 & 7 & 8 & 27 \\
Dupla mensagem & 17 & 4 & 19 & 40 \\
\hline TOTAL & $\mathbf{4 4}$ & $\mathbf{4 8}$ & $\mathbf{7 4}$ & $\mathbf{1 6 6}$ \\
\hline
\end{tabular}

$\mathrm{Na} 1^{\text {a }}$ fase, predominam os modos de dupla mensagem e desconfirmação, o que pode ser compreendido por estarem os alunos nas atividades do primeiro estágio, quando a ansiedade e a insegurança poderiam explicar esses modos de comunicação. 
$\mathrm{Na} 2^{\mathrm{a}}$ fase predominam a confirmação e a negação. Comparando-se esses dados com os da Tabela 1, observa-se que, nesta fase, a ansiedade e a dúvida são menores, em relação a $1^{\mathrm{a}}$ fase.

$\mathrm{Na} 3^{\mathrm{a}}$ fase, aumentam significativamente os números referentes à confirmação e a dupla mensagem paralelamente ao aumento dos números referentes a ansiedade e a dúvida, o que aparece na Tabela 1.

Esses dados mostram que os alunos na $1^{\text {a }}$ fase usam muitas vezes a dupla mensagem e a desconfirmação. $\mathrm{Na} 2^{\mathrm{a}}$ fase, a confirmação e a negação predominam. Podemos, nesta evolução, considerar que os alunos, ao dominarem melhor as técnicas e se familiarizarem com os pacientes, passam a usar os modos mais adequados de comunicação. $\mathrm{Na}^{\mathrm{a}}$ fase, usam muito a confirmação; porém ocorre aumento imprevisto da dupla mensagem, a qual aparece em momentos das interações onde os alunos manifestam sentimentos de ansiedade. Os alunos confirmam os dados detectados pela pesquisadora nas entrevistas após as interações e nos seus relatos das interações, explicando o seu sentimento de ansiedade pelo fato de não poderem dizer as pacientes o seu prognóstico fechado. Em tais situações, têm a percepção da utilização da dupla mensagem e de como o seu comportamento não foi o mais adequado às necessidades das pacientes.

No exemplo abaixo, descrevemos uma interação com a finalidade de ilustrar o referido:

"Aluna ouve a paciente com atenção".

Paciente descreve a triagem quando chegou, no dia anterior. No momento chegava gente ensangüentada. Não pode ver essas coisas. Diz que estava a ponto de desmaiar. Paciente sorri. Refere-se a cirurgia que vai fazer.

Aluna aparenta preocupação. Sorri, retribuindo o sorriso da paciente. - E, vamos ver. Acho que vai ser rápido. Muitas pessoas já fizeram essa cirurgia.

A paciente diz que nunca deixaria operar a cabeça. Conta sobre a cirurgia da cunhada.

- E esta cirurgia você já viu alguém que fez?

A paciente diz que sim, a da vizinha do leito ao lado. Diz que gostaria de saber o que vai Ihe acontecer na cirurgia.

A aluna ouve atenta, olhando para ela.

— Você quer falar mais alguma coisa?

- Deixa para outra hora.

"A aluna diz que está aí para falar com ela".

Com relação a esta interação, a aluna escreve no seu relato: "Fiquei um pouco em silêncio com relação à cirurgia, foi ela quem falou mais, pois senti um pouco de ansiedade, sem saber o que falar, por não ter certeza da cirurgia a que será submetida". 
Após a entrevista com a aluna, avaliamos com ela a necessidade de voltar a conversar com a paciente, ajudando-a a expressar os seus receios, com o objetivo de diminuir a sua ansiedade. A aluna propôs-se a procurar informações sobre o diagnóstico e a cirurgia da paciente para poder dar-Ihe informações precisas.

TABELA 3 - DISTRIBUIÇÃO DAS COMUNICAÇÕES TERAPÊUTICAS E NÃOTERAPÊUTICAS UTILIZADAS PELOS ALUNOS DURANTE AS INTERAÇÕES COM OS PACIENTES. BOTUCATU, 1992

\begin{tabular}{lcccc}
\hline \multicolumn{5}{c}{ FASES } \\
\hline COMUNICAÇÕES & $\mathbf{1}^{\mathbf{a}}$ & $\mathbf{2}^{\mathbf{a}}$ & $\mathbf{3}^{\mathbf{a}}$ & TOTAL \\
\hline Terapêutica & 80 & 144 & 174 & 398 \\
Não-Terapêutica & 112 & 70 & 68 & 250 \\
\hline TOTAL & $\mathbf{1 9 2}$ & $\mathbf{2 1 4}$ & $\mathbf{2 4 2}$ & $\mathbf{6 4 8}$ \\
\hline
\end{tabular}

Com relação à distribuição da comunicação terapêutica, observamos, pelos dados da Tabela 3, que os alunos passaram a usá-la mais na $2^{\mathrm{a}}$ e na $3^{\mathrm{a}}$ fase, o que para nos correspondeu ao desenvolvimento da habilidade em comunicar-se. Inversamente, a comunicação não terapêutica aparece menos vezes no transcorrer das rases, dados que sugerem a percepção e o controle melhor da comunicação não terapêutica por parte dos alunos.

Nas duas tabelas seguintes, n. ${ }^{\circ} 4$ e n. ${ }^{\circ} 5$, passamos a identificar, respectivamente, as estratégias de comunicação terapêutica e as modalidades da não terapêutica utilizadas pelos alunos.

TABELA 4 - DISTRIBUIÇÃO DAS ESTRATÉGIAS DE COMUNICAÇÃO TERAPÊUTICAS UTILIZADAS PELOS ALUNOS DURANTE AS INTERAÇÕES COM OS PACIENTES. BOTUCATU, 1992

FASES

\begin{tabular}{lcccc}
\hline \multicolumn{1}{c}{$\begin{array}{c}\text { ESTRATÉGIAS DE COMUNICAÇÃO } \\
\text { TERAPÊUTICAS }\end{array}$} & $\mathbf{1}^{\mathbf{a}}$ & $\mathbf{2}^{\mathbf{a}}$ & $\mathbf{3}^{\mathbf{a}}$ & TOTAL \\
\hline Ouvir reflexivamente & 3 & -- & 11 & 14 \\
Verbalizar aceitação & 8 & 14 & 9 & 31 \\
Verbalizar interesse & 23 & 21 & 30 & 74 \\
Repetir comentários do paciente & 1 & -- & 2 & 3 \\
Fazer perguntas & 22 & 49 & 62 & 133 \\
Introduzir problema relacionado & -- & -- & 1 & 1 \\
Usar frases descritivas & 23 & 60 & 57 & 140 \\
Verbalizar dúvida & -- & -- & 2 & 2 \\
\hline TOTAL & $\mathbf{8 0}$ & $\mathbf{1 4 4}$ & $\mathbf{1 7 4}$ & $\mathbf{3 9 8}$ \\
\hline
\end{tabular}


Observarmos, pelos dados acima, que as estratégias Fazer perguntas, Usar frases descritivas e Verbalizar interesse estão situadas entre aquelas mais utilizadas pelos alunos, nas três fases. Ouvir reflexivamente aparece mais na $3^{\text {a }}$ fase. Podemos supor, nesta fase que os alunos, já despreocupados da execução das técnicas, puderam colocar como objetivo da interação desenvolver comunicação mais efetiva com os pacientes.

TABELA 5 - DISTRIBUIÇÃO DAS MODALIDADES DE COMUNICAÇÃO NÃO TERAPÊUTICA UTILIZADAS PELOS ALUNOS DURANTE AS INTERAÇÕES COM OS PACIENTES. Botucatu, 1992

\begin{tabular}{lcccc}
\hline & \multicolumn{3}{c}{ FASES } \\
\hline COMUNICAÇÃo NÃO TERAPÊUTICA & $\mathbf{1}^{\mathbf{a}}$ & $\mathbf{2}^{\mathbf{a}}$ & $\mathbf{3}^{\mathbf{a}}$ & TOTAL $^{\text {(14 }}$ \\
\hline Não saber ouvir & 14 & 9 & 6 & 29 \\
Usar termos com conotação de julgamento & - & - & 1 & 1 \\
Induzir respostas & 17 & 8 & 7 & 32 \\
Dar conselhos & - & 2 & 1 & 3 \\
Introduzir respostas não relacionadas & 5 & 3 & 4 & 12 \\
Colocar o paciente à prova & 1 & 2 & 1 & 4 \\
Permanecer na defensiva & - & 1 & - & 1 \\
Comunicar-se unidirecionalmente & 25 & 17 & 10 & 52 \\
Frases estereotipadas & 14 & 16 & 9 & 39 \\
Bateria de perguntas & 7 & 1 & 2 & 10 \\
Desconfirmação & 12 & 7 & 8 & 27 \\
Dupla mensagem & 17 & 4 & 19 & 40 \\
\hline TOTAL & $\mathbf{1 1 2}$ & $\mathbf{7 0}$ & $\mathbf{6 8}$ & $\mathbf{2 5 0}$ \\
\hline
\end{tabular}

Observando os dados da tabela acima, entre as modalidades da comunicação não terapêutica mais utilizadas pelos alunos, aparecem comunicar-se unidirecionalmente, dupla mensagem, frases estereotipadas, induzir respostas.

Entendemos que comunicar-se unidirecionalmente pode representar uma característica, freqüente na comunicação dos alunos devido a natureza da assistência de enfermagem existem regras, normas, horários que são impostos aos pacientes e, via de regra, estes não são consultados e não participam das decisões desses procedimentos. Como durante as interações os alunos desenvolveram procedimentos com horário determinado, esses momentos de comunicação unilateral parecem-nos corresponder às situações citadas. A preocupação do aluno com os próprios objetivos muitas vezes leva a esta modalidade de comunicação, bem como ao não saber ouvir que associamos a ansiedade dos alunos com a execução dos procedimentos nesta fase. 
Os comentários sobre o uso da dupla mensagem devido à ansiedade manifestada e confirmada pelos alunos encontra-se na analise das Tabelas 1 e 2.

\section{TABELA 6 - DISTRIBUIÇÃO DAS VARIÁVEIS IDENTIFICADAS NAS INTERAÇÕES ALUNO-PACIENTE. Botucatu, 1992}

\begin{tabular}{lcccc}
\hline & \multicolumn{5}{c}{ FASES } \\
\hline VARIÁVEIS & $\mathbf{1}^{\mathbf{a}}$ & $\mathbf{2}^{\mathbf{a}}$ & $\mathbf{3}^{\mathbf{a}}$ & TOTAL \\
\hline Contexto & 10 & 9 & 10 & 29 \\
Insegurança do aluno com as técnicas & 2 & 2 & 1 & 5 \\
Ansiedade do aluno & 9 & 8 & 9 & 26 \\
Pouca disponibilidade do aluno & 5 & 2 & 2 & 9 \\
Ansiedade do paciente & 5 & 8 & 9 & 22 \\
Pouca disponibilidade do paciente & 2 & 2 & - & 4 \\
\hline TOTAL & $\mathbf{3 3}$ & $\mathbf{3 1}$ & $\mathbf{3 1}$ & $\mathbf{9 5}$ \\
\hline
\end{tabular}

Observa-se nesta tabela que as variáveis que mais interferiram na comunicação foram ansiedade do aluno, variáveis de contexto e ansiedade do paciente.

Em todas as comunicações aluno-paciente observamos variáveis que interferiram na interação, o que foi percebido e relatado pelos alunos na entrevista. As variáveis de contexto foram citadas por todos os alunos. E compreensível que tenha havido esta interferência dadas as situações onde se realizaram as interações: eram locais de estagio, num hospitalescola onde os alunos desenvolviam atividades diárias. Não houve a preocupação de "isolar" as situações de estudo. A ansiedade do aluno e a ansiedade do paciente aparecem em função das variáveis do contexto: maior ou menor gravidade do estado do paciente, conhecimento da situação do paciente, condições do aluno em prestar assistência adequada. A ansiedade dos alunos de acordo com os dados obtidos, sempre foi percebida pelos alunos; a ansiedade dos pacientes foi sendo mais percebida, nos parece, a medida que os alunos deixaram de se preocupar com as técnicas de enfermagem e de comunicação, tornaram-se mais seguros e conseguiram olhar "para fora de si"- para o paciente. Esta é uma observação que mereceria investigação mais minuciosa e conclusiva. 


\section{TABELA 7 - DISTRIBUIÇÃO DA PERCEPÇÃO DOS ALUNOS A RESPEITO DA INTERAÇÃO DESENVOLVIDA COM O PACIENTE. Botucatu, 1992}

\begin{tabular}{lllll}
\hline \multicolumn{1}{c}{ FASES } & & & & \\
\hline PERCEPÇÃo REFERIDA PELOS ALUNOS & $\mathbf{1}^{\text {a }}$ & $\mathbf{2}^{\text {a }}$ & $\mathbf{3}^{\text {a }}$ & TOTAL \\
\hline $\begin{array}{l}\text { Preocupação e/ou dificuldade com as técnicas de } \\
\text { enfermagem }\end{array}$ & 6 & - & - & 6 \\
Percepção das variáveis & 10 & 6 & 9 & 25 \\
$\begin{array}{l}\text { Percepção dos modos de comunicação não } \\
\text { terapêuticos }\end{array}$ & 6 & - & 4 & 10 \\
Percepção do uso das técnicas terapêuticas & 7 & 2 & 4 & 13 \\
Percepção do uso das técnicas não terapêuticas & 6 & 4 & 5 & 15 \\
Percepção de ter atingido o objetivo da interação & 4 & 6 & 6 & 16 \\
Percepção da própria ansiedade & 3 & 6 & 9 & 18 \\
Percepção da ansiedade do paciente & 2 & 2 & 6 & 10 \\
Percepção da dificuldade em comunicar-se & 4 & 4 & 2 & 10 \\
\hline TOTAL & 48 & 30 & 45 & 123 \\
\hline
\end{tabular}

Em relação à própria percepção, os alunos na $1^{\text {a }}$ fase mostraram maior preocupação com as técnicas de enfermagem, com as variáveis que interferiram na comunicação, com as técnicas terapêuticas, os modos não terapêuticos e as técnicas não terapêuticas. Os alunos na $2^{\mathrm{a}}$ fase mostraram preocupação maior com as variáveis, a própria ansiedade, ter atingido o objetivo da interação, as técnicas não terapêuticas e a dificuldade em comunicar-se. Os alunos na $3^{a}$ fase referiram preocupação maior com a própria ansiedade, as variáveis que interferiram na comunicação, a ansiedade do paciente, ter atingido o objetivo da interação, e as técnicas não terapêuticas. Percebemos, como dados significativos, a evolução dos alunos da $1^{\mathrm{a}}$ para a $2^{\mathrm{a}}$ e para a $3^{\mathrm{a}}$ fase em relação a percepção da própria ansiedade e da ansiedade do paciente. Em contrapartida, ocorre o inverso em relação a percepção das técnicas terapêuticas e das técnicas não terapêuticas. Podemos supor que a preocupação possa ser mais quando não se consegue usá-las usando-as ocorre uma certa despreocupação e tornam-se de certo modo integradas a sua pratica de modo inconsciente. Passamos a comparar trechos de interações das 3 fases, procurando ilustrar como a ansiedade interferiu na comunicação dos alunos com o paciente, assim como na utilização das medidas e técnicas terapêuticas. 


\section{Exemplo da $1^{\text {a }}$ FASE}

"Aluna pergunta se a paciente está bem. (olha para ela).

Paciente responde que sim. (Paciente está sentada numa cadeira).

Aluna pede licença para colocar o termômetro. Coloca-o. Começa a verificar o pulso do mesmo lado, meio desajeitada e confusa. Pergunta a paciente se o braço dói.

A paciente diz que "parece doer as veias".

Aluna muda de lado pega o outro pulso. Demonstra expressão de dúvida.

A aluna murmura para si mesma. "Está bem baixinho". Balança a cabeça.

Continua contando o pulso, compenetrada.

A paciente observa a outra paciente, rodeada de médicos. Observa a aluna. Sorri.

A aluna observa o abdômen da paciente (conta a respiração). Pede ajuda a colega. Ajoelhase para contar o pulso. Diz a ela: "É bem baixinho". Fica de pé. A outra aluna ajoelha-se ao lado da colega e verifica o pulso. (Confabulam as duas alunas). A aluna anota e observa a paciente. Uma funcionaria traz a medicação. A paciente toma e faz careta".

Nesta situação, da $1^{\mathrm{a}}$ fase, a aluna parece muito preocupada com a verificação dos sinais, o que ela confirma na entrevista. Refere que "foi ficando ansiosa por que não conseguia achar o pulso e não conseguia contar". Avaliou que a paciente estava calma. Seu objetivo volta-se para o desenvolvimento da técnica de verificação dos sinais vitais, a ansiedade não Ihe permitindo dar atenção a paciente.

\section{Exemplo da $2^{\mathrm{a}}$ FASE}

"Paciente em cadeira de rodas. Não fala (teve um AVC). Acabou de tomar o café.

A aluna tira a xícara do café. Olha para a paciente. - M. vamos tomar medicação.

Paciente assente com a cabeça.

Aluna sorri. - M., aqui (oferece os comprimidos). Um remédio para o coração e um para fazer xixi. Vai engolir os dois de uma vez?

Paciente pega os comprimidos.

Aluna oferece água a paciente. - Engoliu? (olha atenta para a paciente).

Tudo bem?

Paciente balança a cabeça que sim. Engole os comprimidos, bebe a água.

Aluna olha atenta para a paciente. - Quer mais alguma coisa?

Paciente faz sinais com a mão e expressão no rosto.

A aluna sorri. - Vamos para a cama agora, M. ?

Paciente faz um grunhido assentindo. Olha atentamente para a aluna. 
A aluna aproxima-se da paciente".

Em relação a esta interação, na $2^{\mathrm{a}}$ fase, a aluna descreve no seu relato "a preocupação em não induzir, conseguir detectar as necessidades da paciente sem induzir", devido ao rato da paciente não ralar. A aluna parece, aqui, muito atenta e preocupada em conseguir diagnosticar as necessidades da paciente através de uma comunicação eficiente. Seu objetivo volta-se para a paciente.

\section{Exemplos da $3^{\mathrm{a}}$ FASE}

"Paciente parece inquieta. Pré-operatório de cirurgia da mama (biopsia).

Paciente diz que já vieram arrumar a cama: - Como aqui não tem doença infecciosa, não precisa trocar.

Aluna ouve atenta. - No hospital troca-se a roupa todo dia.

A paciente queixa-se que foi acordada a noite de um modo brusco, e que fica com vontade de bater na pessoa que a acorda assim.

A aluna ouve a paciente, olhando-a, com atenção. - Você acha que, se tivesse sido acordada de outro modo, você teria outra reação?

A paciente responde que sim.

- O que a gente pode fazer e deixar anotado sobre isso para não acontecer novamente".

Em relação a esta situação, numa interação da $3^{a}$ fase, observa-se a atenção da aluna ouvindo as queixas da paciente, percebendo a sua ansiedade, o que a aluna relata na entrevista. Neste trecho da interação, a aluna ouve reflexivamente, utiliza pergunta e sugere uma solução para a questão (frase descritiva). Em resumo, oferece apoio a paciente, tendo percebido a sua ansiedade. Seu objetivo volta-se para atender as necessidades da paciente, ajudando-a a resolver uma questão que a incomoda.

Mais adiante, na mesma interação, a aluna mostra-se ansiosa e isso parece no registro da interação (confirmado por ela na entrevista), interferindo na sua comunicação com a paciente.

"Paciente rala continuamente sobre a sua cirurgia: fala sobre a possibilidade de ser extraída a mama, sobre a autorização para a extração, sobre a cirurgia. Que a médica vai abrir e ver se precisa tirar o seio. Fala precipitadamente.

Aluna ouve atentamente. Expressão tensa desvia o olhar. Explica a paciente as várias possibilidades. - Não e importante só para você, e para todo mundo. Depois da cirurgia pode usar um sutiã até ter a prótese... (Pausa).

A paciente diz que sempre quis fazer plástica no seio e o marido não deixou.

A paciente diz achar que se tivesse feito, talvez não tivesse esse problema.

- A sua médica, era da cidade? (olha para a paciente). 
Paciente explica sobre as duas médicas que a atenderam. Diz que foi a médica já com o caroço, que havia percebido fazia algum tempo, com dores. A aluna tira o termômetro, verifica, anota na papeleta e continua conversando. - Nessa época não relatou isso a médica? (lava o termômetro na pia)".

$\mathrm{Na}$ mesma interação do exemplo anterior, na qual oferecia apoio a paciente, no momento em que esta começou a falar do seu problema central - a retirada da mama, a aluna passou a utilizar técnicas não terapêuticas: frases estereotipadas e mudança de assunto. E, pelo seu não-verbal, dupla mensagem (o que ela confirmou na entrevista). A aluna descreve no relato: "ela queria respostas que eu não conseguia dar". A ansiedade da aluna levou-a ao uso de comunicação não terapêutica.

Ao apresentar estes exemplos de trechos de interação aluno-paciente, nosso objetivo foi ilustrar como a ansiedade das alunas interferiu na utilização da comunicação terapêutica, em decorrência disso prejudicando a percepção das necessidades do paciente e a própria assistência integral de enfermagem. Consideramos, nestas situações, que o conhecimento da teoria da comunicação terapêutica permitiu que as alunas percebessem a própria ansiedade, assim como a ansiedade dos pacientes.

\section{CONSIDERAÇÕES FINAIS}

Os resultados obtidos neste estudo mostram, de um lado, a evolução do uso da comunicação terapêutica pelos alunos ao prestarem assistência ao paciente. For outro lado, mostram o sentimento de ansiedade como uma variável importante interferindo no relacionamento aluno-paciente, conseqüentemente no próprio cuidado prestado. A evolução no senti do de perceber a própria ansiedade, assim como a percepção da ansiedade do paciente e como esta dificulta a sua comunicação com o paciente parece estar relacionada como o conhecimento e o uso da teoria da comunicação terapêutica. Estes dados sugerem que o desenvolvimento das habilidades em comunicar-se oferece recursos aos alunos para perceberem a ansiedade que sentem ao interagirem com os pacientes em situações críticas, e, uma vez percebida esta ansiedade, elaborem formas de diminuí-la para níveis suportáveis.

Neste sentido, é de fundamental importância reafirmar a necessidade do oferecimento de apoio aos estudantes por parte dos docentes e profissionais envolvidos nas atividades de estágio, com o objetivo de ajudá-los a desenvolver os mecanismos mais adequados para lidarem com a própria ansiedade. 


\section{A STUDY OF ANXIETY AS A VARIABLE IN THE STUDENT-PATIENT RELATIONSHIP}

The aim of this study was to analyze the anxiety as a variable in the nursing students communication with patients. Data was obtained through interviews, reports and observations of 10 students during 3 moments: 3rd, 4th, and 6th, periods of undergraduate nursing course. Both students and patients anxiety was identified as a variable that interfered in the use of the therapeutic communication by the students.

UNITERMS: anxiety, therapeutic communication

\section{ESTUDIO DE LA ANSIEDAD COMO VARIABLE EN EL RELACIONAMIENTO ALUMNOS Y PACIENTES}

El estudio tiene por objetivo el análisis de la ansiedad como variable en la comunicación de los alumnos de graduación de enfermería, en actividades relacionadas a cuidados de pacientes. Los datos fueron obtenidos a partir de la observación participante, estrategia esta que constituida por tres procedimientos simultáneos: registro por la investigadora de las interacciones entre alumnos y pacientes; registro por los alumnos de esas mismas interacciones y entre vista de los alumnos por la autora mediatamente después de los dos primeros procedimientos. La populación se constituyó de 10 alumnos de enfermería acompañados durante sus actividades prácticas curriculares en los $3^{\circ}, 4^{\circ}$ e $6^{\circ}$ semestres del curso básico (enfermería). La ansiedad de los estudiantes como la de los pacientes, aparece como variable interferente en el uso de la comunicación terapéutica entre ellos.

UNITERMOS: ansiedad, comunicación terapéutica

\section{REFERÊNCIAS BIBLIOGRÁFICAS}

01. BAILEY, R. D. Coping with stress in caring. London: Blackwell Scientific Publications, 1985.

02. BIRCH, I. The anxious learners. Nurs.Mirror, v. 8, p.17-24, 1979.

03. COLLIGAN, M., SMITH, M., HORREL, J. Occupational incidence rates of mental health disorders. J.Hum.Stress, v. 3, p. 34-42, 1977. 
04. EDWARDS, B.J, BRILHART, J.K. Communication in nursing practice. Saint Louis Mosby, 1985.

05. HOFLING, C.K. et al. Enfermería psiquiátrica. 2. ed. México: Interamericana, 1970

06.LUDKE, M., ANDRE, M E D.A. Pesquisa em educação: abordagens qualitativas. São Paulo. EPU, 1986.

07. MARSHALL, J. Stress amongst nurses. In COOPER, C., MARSHALL, J. White Collor and Professional Stress. London: Wiley, 1980.

08. MAY, R. O significado da ansiedade: as causas da integração e desintegração da personalidade. Rio de Janeiro: Zahar, 1980.

09. MENZIES, I.E.P. Nurses under stress. Int.Nurs.Rev., v. 7, p. 9-16, 1960.

10. PESSOTTI, I. Ansiedade. São Paulo: EPU, 1978.

11. SADALA, M.L.A Desenvolvendo a habilidade em comunicação. São Paulo, 1992. (Trabalho apresentado ao Congresso Internacional de Pesquisa em Enfermagem, Escola de Enfermagem da USP, São Paulo).

12. STEFANELLI, M.C. Ensino de técnicas de comunicação terapêutica enfermeirapaciente. São Paulo, 163 p. Tese (Doutoramento em Enfermagem) - Escola de Enfermagem da USP, 1985.

13. SILVA, M.J.P. A percepção das enfermeiras sobre a comunicação não verbal dos pacientes. São Paulo, 108 p. Dissertação (Mestrado) - Escola de Enfermagem da USP, 1989. 